\title{
Sobre la inscripción del mosaico helenístico de Ilici (La Alcudia, Elche)
}

\author{
The inscription in the \\ Hellenistic mosaic of Ilici \\ (La Alcudia, Elche)
}

\author{
Ignacio Simón Cornago \\ Università degli Studi di Roma Tor Vergata \\ isimoncornago@gmail.com
}

Resumen: La inscripción musiva de La Alcudia, la ciudad ibérica de Ilici, es excepcional porque es el epígrafe más antiguo realizado sobre opus tessellatum de Hispania y también por ser una de las pocas inscripciones que pueden clasificarse como un texto ibérico escrito en alfabeto latino. El objetivo de este trabajo es proponer una nueva edición y lectura además de realizar algunas observaciones sobre su interpretación a partir de los paralelos latinos.

Palabras clave: Antropónimos ibéricos. Lengua ibérica. Alfabeto latino. Inscripción musiva. Latinización.

Abstract: The mosaic inscription from La Alcudia, the ancient Iberian city of Ilici, is excepcional because it is the most ancient opus tessellatum inscription discovered in Hispania and also because it is one of the few inscriptions that could be classified as an Iberian text written in Latin alphabet. The aim of this paper is to propose a new lecture according to the autopsy and make some remarks on its interpretation according to the Latin parallels.

Key words: Iberian personal names. Iberian language. Latin alphabet. Mosaic inscription. Latinization.

Recepción: 10.09.2019 | Aceptación: 25.11.2019

Proyecto: Este trabajo se ha realizado en el marco de un contrato Marie Sklodowska-Curie de la Unión Europea (Grant agreement 794476) y se inscribe en el proyecto FFI2015-63981C3-1-P, "Hesperia: lenguas, epigrafía y onomástica paleohispánica", financiado por el Ministerio de Economía y Ciencia de España. 


\section{Introducción}

El epígrafe musivo de La Alcudia es bien conocido y singular por varios motivos: es la inscripción realizada en opus tessellatum más antigua de Hispania y se ha reconocido como uno de los escasos textos redactados en lengua ibérica y alfabeto latino. ${ }^{1}$ Su lectura está comprometida por el estado de conservación del mosaico. En este trabajo se propone una nueva lectura y edición que permiten avanzar en la interpretación de este complejo texto.

\section{Historia del descubrimiento y ediciones}

El mosaico fue descubierto en el año 1959 y publicado por Ramos 1975, en la revista Archivo de Prehistoria Levantina, en un artículo monográfico en cuyo título se califica como "mosaico helenístico", término con el que suele conocerse en la bibliografía especializada (fig. 1). Fue exhumado en una habitación del sector 5-F, en el nordeste del yacimiento, que forma parte de una vivienda que también se denomina 5-F y de la que se conoce mejor su fase altoimperial (Sarabia y Cañavete 2010). Del periodo anterior hay tres estancias contiguas: una pavimentada con opus signinum, otra con un pavimento de adobes cubiertos de cal y la tercera, que es la de mayor tamaño, acoge el mosaico inscrito y se ha reconocido como una estancia de aparato, habiéndose clasificado como el tablinum de la casa (Abad 2004, 76).

La inscripción está escrita en alfabeto latino, pero es cuestionable que también pueda interpretarse como un texto latino. Siles realizó en 1978 el primer estudio lingüístico del epígrafe y lo interpretó como un texto ibérico escrito en alfabeto latino, clasificación que han mantenido, con o sin matices, los editores posteriores. ${ }^{2}$ El epígrafe ha sido compilado en varios corpora: Untermann lo recoge en el tercer volumen de $M L H$ (G.12.4) -ahora también está editado en el Banco de Datos Hesperia (BDH A.10.04) - y Gómez Pallarès en su catálogo de las inscripciones sobre mosaico de Hispania (ECIMH A1); también se recoge, dado que está escrito en alfabeto latino, en diferentes corpora de epigrafía romana: el de Rabanal y Abascal sobre la epigrafía de Alicante $\left(1985, \mathrm{n}^{\circ}\right.$ 96) y los de Corell sobre Ilici $(1999,2012)$.

1 Sobre la epigrafía ibérica de La Alcudia: $M L H$ III G.12.

2 La única excepción es D’Ors 1960, 328, que, a partir de fotografía facilitada por A. Ramos, propone una interpretación latina errada: Satu(?) Acos / et Sabini Cor(nelii) / $\operatorname{scrad}(?)[\ldots] / /$ Acos. 


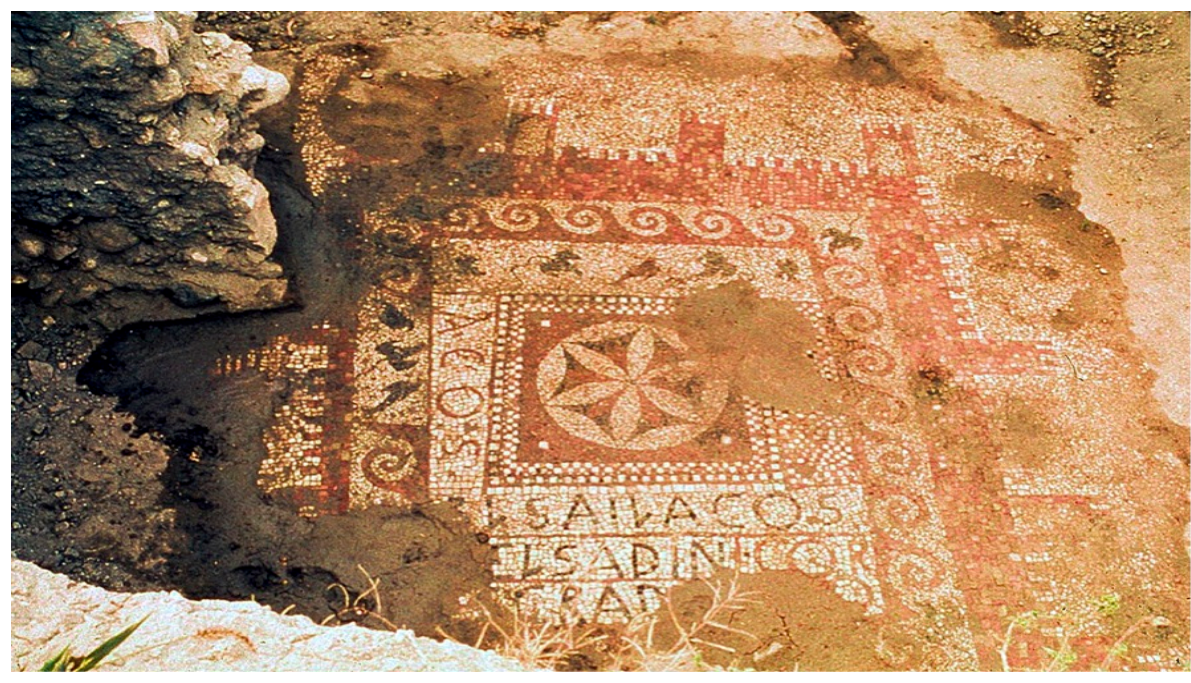

Fig. 1. Fotografía del mosaico en el momento del descubrimiento (foto: Fundación La Alcudia).

\section{Descripción e iconografía}

El pavimento combina el uso de guijarros y teselas (opus tessellatum), empleándose piezas de piedra y también de cerámica, por todo ello Abad 198687, 101-102 califica de "mixta" la técnica con la que se ejecutó este pavimento (fig. 2). ${ }^{3}$ También destaca la policromía, empleándose teselas negras, azules, blancas, castañas y rojas. La inscripción está realizada en dos colores: rojo y gris azulado. Las teselas rojas se emplean para la palabra que aparece separada del resto del epígrafe (ACOS) y también en las últimas letras de la tercera y cuarta líneas. En este periodo no es común la bicromía en las inscripciones musivas y en las que se emplea se alternan cuidadosamente teselas de diferentes colores en cada una de las letras con claros fines estéticos, una pretensión que no se aprecia en el ejemplar de La Alcudia. ${ }^{4}$

El centro del pavimento está ocupado por una roseta hexapétala inscrita en un círculo que, a su vez, está delimitado por un cuadrado de teselas de color castaño. En torno al cuadrado hay una banda de ajedrezados en los que alternan piezas blancas y marrones. La simetría se rompe en este punto pues la inscripción ocupa dos lados, otra banda de ajedrezado el tercero y varios

3 Vid. tb. Ruiz 2001, 32-34. Sobre el origen del mosaico de guijarros en la península Ibérica: Fernández-Galiano 1982.

4 Inscripciones de Marsella (Vassal 2006, n 103) y Pompeya, ante la casa del Fauno (CIL $\mathrm{X} 872$ ). 
elementos decorativos realizados con teselas negras cubren el cuarto. Engloba todo el conjunto una banda con postas rojizas que solo en las esquinas se ven interrumpidas por hojas de vid ejecutadas con teselas negras y, bajo el epígrafe ACOS, por dos delfines que flanquean una quinta hoja. La última cenefa recorre, al menos, tres de los lados y se compone de murallas con torres. ${ }^{5}$ La mayor parte de los elementos iconográficos, roseta, postas, delfines, hojas de parra, murallas torreadas, están ampliamente documentados en otros mosaicos de época helenística, ${ }^{6}$ la mayoría aparecen en los mosaicos de opus tessellatum delienses que se fechan en torno al año 100 a.C. (ajedrezados, postas, murallas, rosetas, delfines, Bruneau 1972, 46-51 y 69-70); las rosetas inscritas en un círculo y los delfines también son comunes en los opera signina coetáneos (Vassal 2006, 52-57).

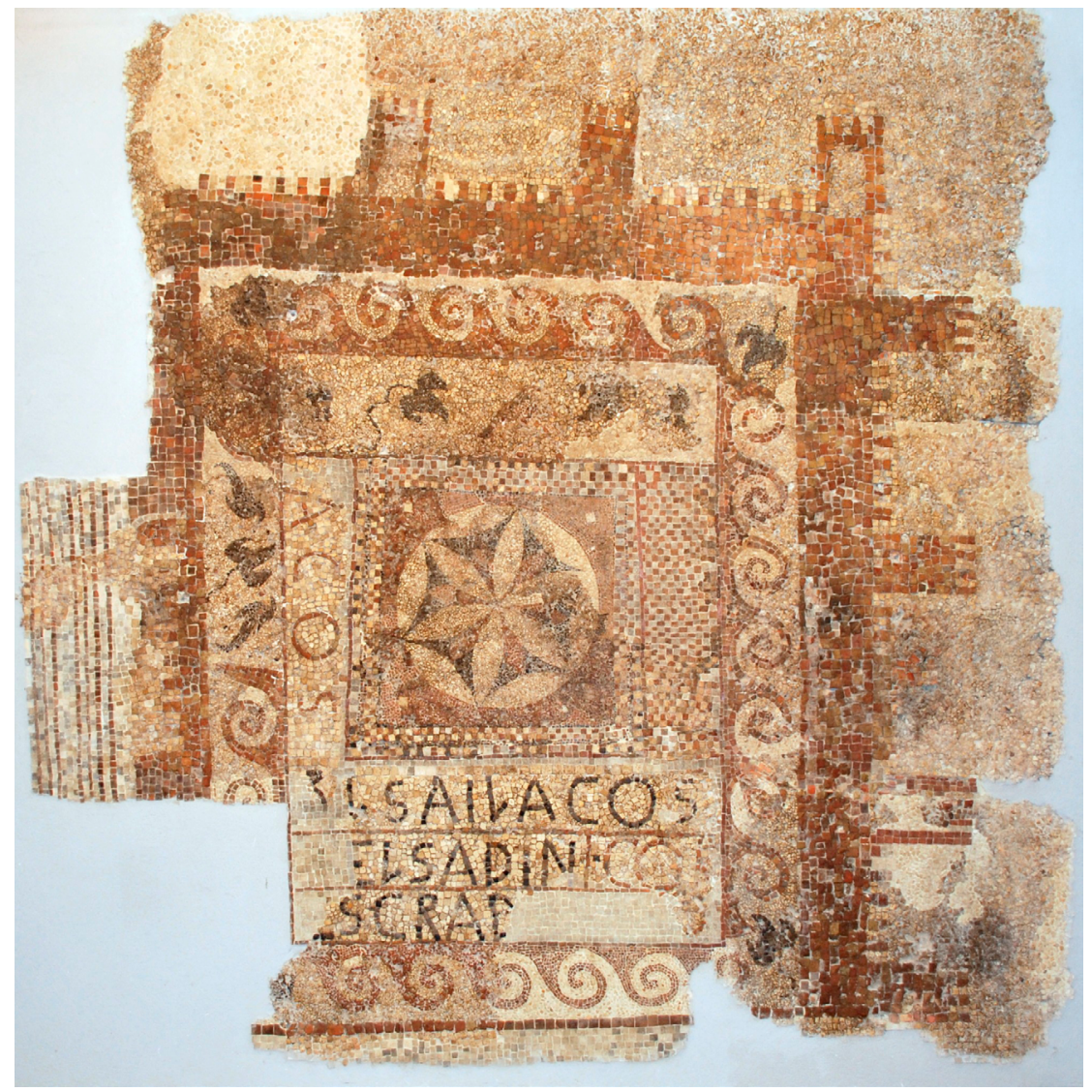

Fig. 2. El mosaico helenístico de Ilici (foto: Fundación La Alcudia).

5 Sobre este elemento en la musivaria hispana, véase el trabajo de San Nicolás 2004.

6 Vid. Abad 1987, 102-102. 
Debe destacarse que los animales que se representan no son pájaros sino delfines, contrariamente a lo que han defendido todos los autores, tanto Ramos 1975, 73, como Abad 1986-87, 101, y también Tammisto 1997, CAT.SC10, en su monografía sobre las representaciones de aves en mosaicos de época helenística. Los delfines son comunes en los mosaicos y suelen aparecen asociados a las postas. ${ }^{7}$ En los opera signina de Hispania aparecen en Caminreal, Mataró, Badalona y Cartagena, en los ejemplares de la calle Soledad y en el del Faro del Estacio (Fernández 2003, fig. 5, 10, 12, 19, 21; uid. tb. Ramallo 1985, 62-63). Pueden señalarse algunos paralelos desde el punto de vista iconográfico. El primero es el pavimento del caldarium de las termas de Musarna (Etruria), fechado a finales del siglo II a.C. y en el que se combina opus figlinum y opus tessellatum (fig. 3).

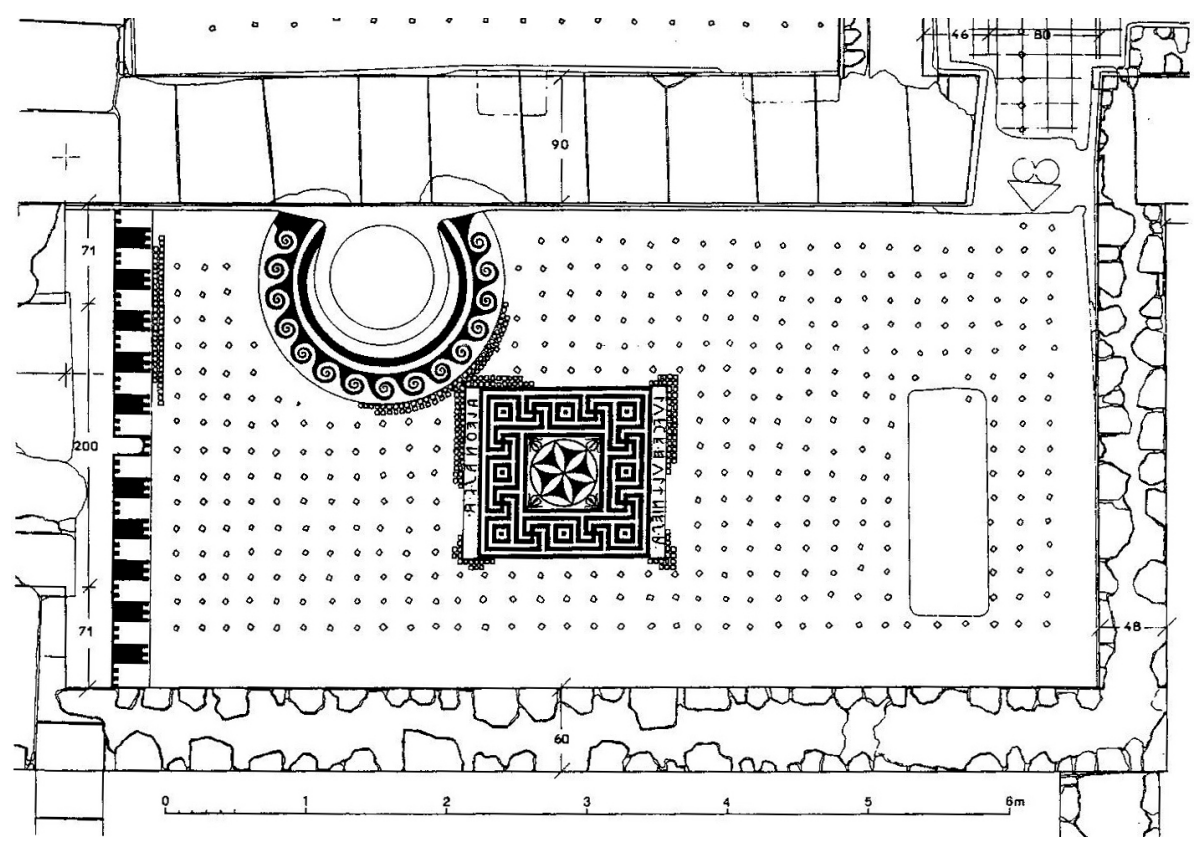

Fig. 3. Pavimento de las termas helenísticas de Musarna, Etruria (sg. Broise y Jolivet 2004).

7 Está documentada en los pavimentos de guijarros como los de Arpi, fechados en la segunda mitad del siglo III a.C. (Salzmann 1982, n. ${ }^{\circ}$ 12-14); también se atestigua en la pintura etrusca, por ejemplo, en la tumba del “Tifone” de Tarquinia, de época helenística (Cristofani 1969, 221, lám. 3). 
Este ejemplar comparte con el ilicitano parte de los motivos iconográficos: una roseta como emblema central, líneas de postas y murallas con torres, también está inscrito con un texto en etrusco que menciona a dos individuos que se ha considerado serían los magistrados que realizaron la obra (ale $\theta$ nas.v.a, luvce.ul nies.a., Broise y Jolivet 2004, 80-91). El segundo paralelo es el mosaico del sacellum del santuario de Hércules Curino en Sulmo, un opus tessellatum polícromo (téseras blancas, negras y rojas) que se fecha antes del Bellum Sociale y en el que también aparece la roseta como elemento central rodeada de cenefas de postas, murallas, delfines y hojas de hiedra (Salcuni 2013, SULMO 01). Las rosetas como elemento central también se utilizan en dos pavimentos de Taranto, uno recuperado en Via Manzzini y el otro en Via Dante Alighieri, en el primero de ellos está rodeada de postas y flanqueada por delfines y se usan, como en el ejemplar de La Alcudia, teselas y guijarros, se fecha a fines del siglo III o comienzos del II a.C. (Dell'Aglio y Masiello 2004).

\section{Datación y paleografía}

Las cronologías propuestas para este mosaico oscilan entre el siglo II y el I a.C. ${ }^{8}$ Ramos 1975, 74-81, indica que proviene del denominado estrato D, que corresponde a finales del siglo I a.C. y, por tanto, fecha el pavimento en la segunda mitad de dicha centuria. ${ }^{9}$ La reciente revisión de los materiales arqueológicos apunta a una fecha ante quem de mediados del siglo I a.C. para el estrato superior y una cronología del siglo I a.C. para los recuperados en el estrato inferior (Lara 2007, 164). No obstante, la técnica y la paleografía presentan algunos elementos arcaizantes que apuntan al siglo II a.C. Abad 1987, que estudió la técnica y la iconografía, se inclina por fecharlo en el siglo II o comienzos del I a.C. Desde el punto de vista de la paleografía destaca la forma de $\mathrm{C}$, muy abierta, ${ }^{10}$ la $i$ longa ${ }^{11}$ y especialmente la $\mathrm{L}$ de tipo calcídico, pues se trata de un rasgo claramente arcaico (fig. 4). Este último elemento,

8 Vid. Lara 2007, 156.

9 Sobre los problemas que plantea la estratigrafía de La Alcudia: Abad 2004, Moratalla 2004-05, Tendero y Ronda 2014.

10 En una inscripción musiva pompeyana con la datación consular del año 59 a.C. aún se utiliza este tipo de C abierta (De Simone 2001), mientras que en otra de Viña Codini con la pareja de cónsules del año 1 d.C. se utiliza ya una C más cerrada (CIL VI 5531).

11 La inscripción datada más antigua en la que aparece es del año 111 a.C. (CIL $\left.\mathrm{I}^{2} 585\right), c f$. Oliver 1966. Se atestigua en varias inscripciones republicanas de Hispania: ELRH U56 (Valdeinfierno, Jaén) y ELRH U63 (Cástulo), está última está parcialmente escrita en latín y parcialmente en una lengua indígena, presumiblemente ibérico (MLH III, H.6.1). 
cuya presencia han subrayado Ramos y Uroz 1992, 98, les lleva a proponer una datación del siglo II a.C. para el epígrafe, pues según señalan, Coarelli 1976, 160, estima que el uso de esta forma no puede ser posterior a los primeros decenios del siglo II a.C. ${ }^{12}$ En la epigrafía republicana de Hispania solo se atestigua en una inscripción punteada sobre un casco recuperado en Pozo Moro (ELRH C54) y en el bronce de Lascuta, fechado en el año 190 o bien en el 189 a. C., años en los que Lucio Emilio Paulo fue pretor en la Hispania Vlterior (ELRH U1 = CIL I ${ }^{2} 614=$ II 5041).${ }^{13}$ En inscripciones musivas aparece en Hatria (CIL I $\mathrm{I}^{2}$ 1895), Luni (CIL I $\left.\mathrm{I}^{2} 3368\right)$, Tibur (CIL I $\mathrm{I}^{2}$ 1496) y la del templo de Apolo Sosiano en Roma (CIL $\left.\mathrm{I}^{2} 2675 \mathrm{c}\right)$, que se han datado todas ellas en el siglo II a.C., excepto la de Tibur que se fecha en el siglo I a.C.

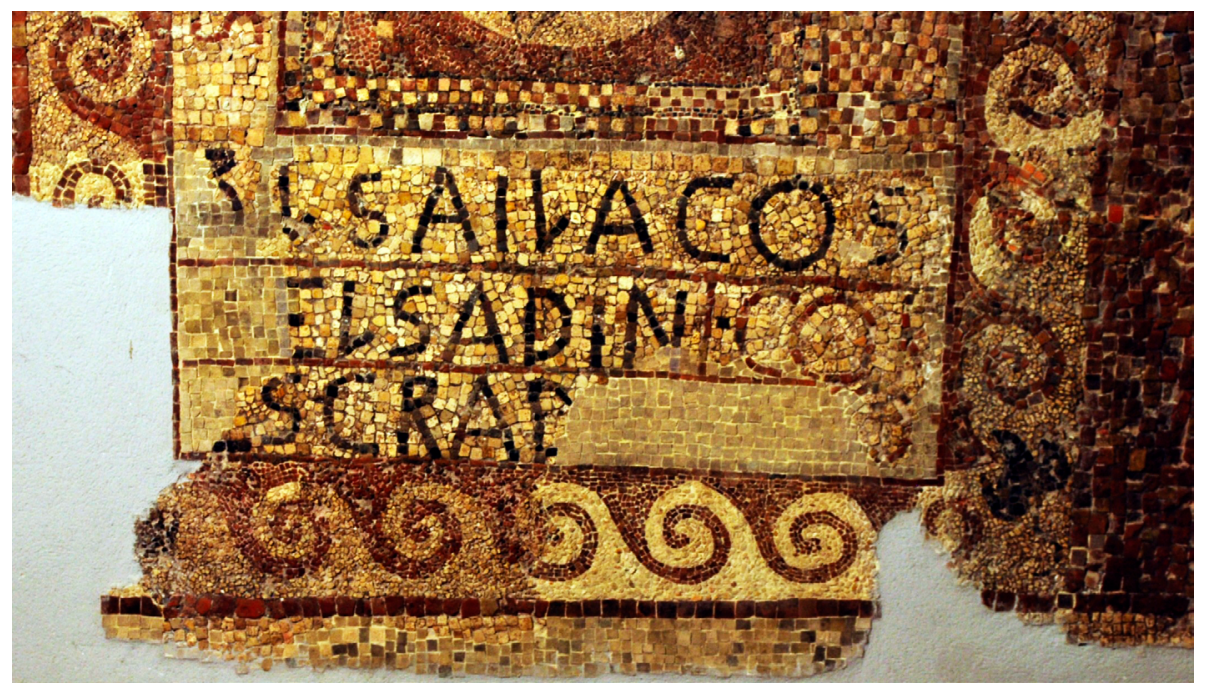

Fig. 4. Detalle de la inscripción del mosaico helenístico de Ilici (foto: Fundación La Alcudia)

12 Crawford en ImIt, p. 14, afirma que las inscripciones en las que aparece no rebasan el 125 a.C.

13 Se documenta igualmente en algunos de los sellos y grafitos latinos más antiguos de Hispania realizados sobre ánforas greco-itálicas, pero que presumiblemente llegaron inscritas desde Italia, $c f . E L R H$, p. 36-38, fig. 1. 


\section{Una inscripción musiva excepcional}

Las inscripciones musivas son una novedad del periodo helenístico. ${ }^{14} \mathrm{En}$ Hispania aparecen en los siglos II y I a.C., entre las que se cuentan ejemplares escritos en tres lenguas: griego (todos recuperados en Ampurias), latín e ibérico. ${ }^{15}$ Están realizados casi exclusivamente en opus signinium, una técnica que se difunde en la región en este momento (Fernández 2003). La inscripción de La Alcudia es el único ejemplar anterior al reinado de Augusto realizado en opus tessellatum. También emplea esta última técnica el pavimento de las termas de Badalona, en el que se conservan unas pocas letras de una inscripción que, como el mosaico, se fecha a inicios del Principado (ECIMH B3). Solo con posterioridad a la edición del ejemplar de La Alcudia se han descubierto otros epígrafes ibéricos sobre opus signinum, son los celebérrimos textos de Caminreal y Andelo (Andión). Los dos están escritos en signario ibérico levantino, proceden del valle del Ebro y además comparten textos muy similares: likinete $\cdot$ ekiar $\cdot$ usekeŕteku (E.7.1 = K.5.3) y likine $\cdot$ abuloŕaune $\cdot$ ekien - bilbiliaŕs (K.28.1). ${ }^{16}$ La interpretación de estos epígrafes no puede abordarse por separado ya que sus textos tienen notables puntos en común. El principal es que ambos están encabezados por el mismo nombre personal: likine. ekiar también aparece en los dos, un término común en las inscripciones ibéricas, y se cierran ambos con un topónimo: usekeŕte seguido de un sufijo -ku en Caminreal (es el nombre de la ciudad ibérica que acuña con leyenda usekerte, A.26) y bilbili seguido del sufijo -aŕs en Andión (el nombre de la ciudad celtibérica que acuña con la leyenda bibilis, A.73).

Su posible clasificación como un texto ibérico redactado en alfabeto latino también lo convierte en un ejemplo excepcional, pues casi no se emplearon las letras romanas para escribir la lengua ibérica. Solo se conocen otros dos posibles epígrafes con estas características, ambos de interpretación igualmente compleja. ${ }^{17}$

14 Sobre la difusión de este tipo de inscripciones: Simón 2018.

15 La epigrafía musiva de Hispania está editada en ECIMH. Para el periodo helenístico: Jaeggi 1999, 169-171.

16 La bibliografía es muy amplia, una recopilación en Simón 2015.

17 H.6.1 (Cástulo) y H.3.4 (Santisteban del Puerto), se podría añadir un breve grafito sobre cerámica recuperado en La Alcudia, $c f$. Simón 2019, con el conjunto de las referencias para estas inscripciones. 


\section{Problemas de lectura e interpretación. Estado de la cuestión}

La lectura está comprometida por el estado de conservación del mosaico que afecta al inicio de las líneas 2, 3 y 4 y también al final de esta última. Una parte de las letras solo se preservan de forma parcial y explica las discrepancias entre los distintos editores; otro punto conflictivo es la existencia o no de una interpunción en el tercer renglón. Respecto a la interpretación, Ramos 1975 señalaba la posibilidad de que el texto recoja antropónimos indígenas: “podría tratarse de nombres iberos latinizados?”, sugerencia que han aceptado todos los editores posteriores. ${ }^{18}$ Fue Siles 1978, quien realizó el primero y casi único estudio lingüístico sistemático. Este autor señala que el texto está escrito en alfabeto latino pero que la lengua no es latín sino ibérico y que el epígrafe solo contiene nombres propios. ${ }^{19}$ Para ACOS indica el paralelo que ofrece la leyenda de una de las dracmas de imitación emporitana: akos (MLH I A.6.02 = $\mathrm{CNH} 35$ n. $^{\circ}$ 84); también recuerda la existencia de un nombre Acco en la Hispania Indoeuropea (Vallejo 2004, 102). Propone reconstruir en la segunda línea [BE]LSAILACOS, que analiza como un nombre personal beleśaiŕ, documentado en el plomo del Solaig (F.7.1), seguido de $a k o s .{ }^{20}$ En la siguiente línea reconstruye [B]ELSADINICOR y lo clasifica como un antropónimo trimembre: belesadin-icor (cf. Faria 1995, 80). En la última línea propone reconocer un nombre personal [I]SC(E)RAD[IN], compuesto por dos formantes bien documentados (iskeŕ y atin, $M L H$ III-1, 7.64 y 19). Untermann (MLH III-2, 614) añade algunos comentarios en su edición del texto. Señala la existencia de un formante onomástico ibérico lakos, pero igualmente la imposibilidad de reconocer otro en la parte que le precede, aunque especula si pudiera ser alsai; en la segunda línea reconoce, como Siles, un nombre personal: [B]ELSADIN; también afirma la posibilidad de leer una $i$ longa tras él, lo que podría ser la desinencia latina de genitivo. Sobre COR estima que pudiera ser un apelativo o bien un Kurzname pues recuerda la existencia de formantes como kori y koro (MLH III-1, 7.78 y 79); y en la última línea también acepta la propuesta de Siles y recuerda la existencia de iskeratin en una leyenda monetal de Obulco (MLH I A.100-4 y 5).

18 Rabanal y Abascal 1985, n 96: "a pesar de la aparente grafía latina, se trata de un texto ibérico cuyo significado se nos escapa". Gómez Pallarés ECIMH, A1: "se trata de nombres ibéricos latinizados". Corell 1999, n. ${ }^{\circ} 24$ : "hom creu que es tracta de noms ibèrics llatinizats".

19 "Zu beachten ist, dass es keine Spuren einer sprachlichen Latinisierung gibt, obwohl der Text in lateinischen Buchstaben geschrieben ist", Siles 1978, 332.

20 Faria 2007; 2018, 121, reconoce OSAILACOS, que interpreta como un NP trimembre. 
30 octulve 1959-2 hour Tarde 77. Se auglia la cata cal mosereaico,

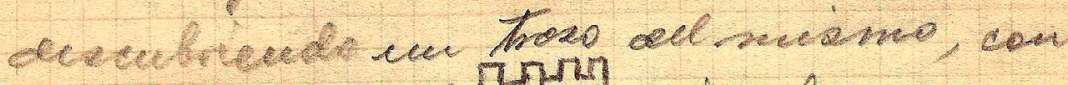

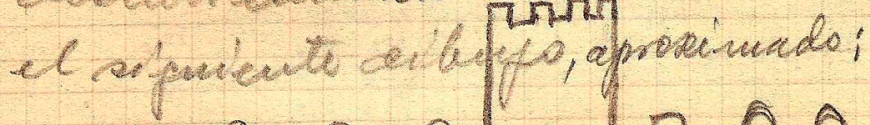
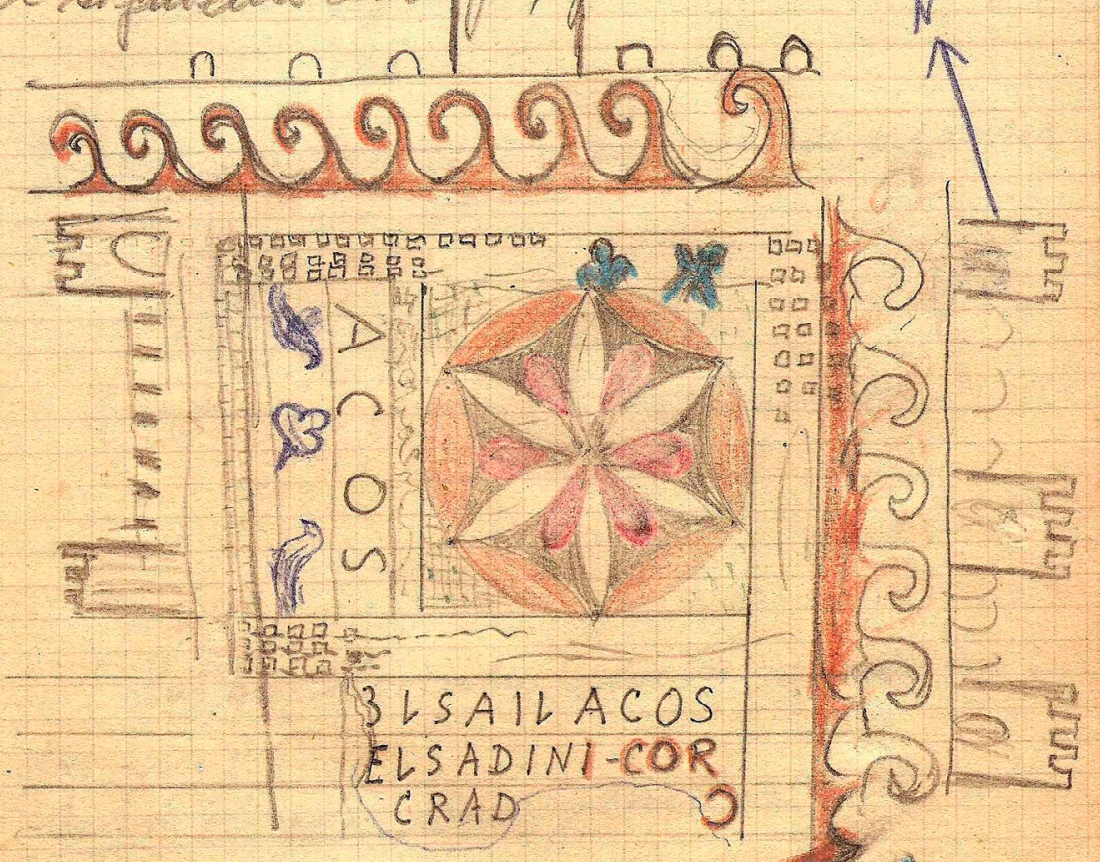

El cceondico esta formado con teseleg pravaly, de

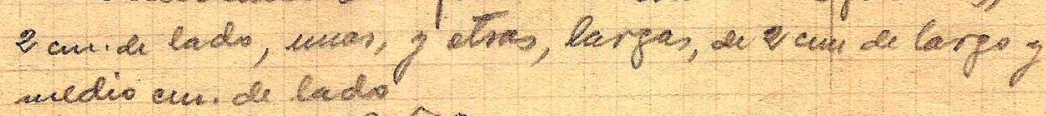

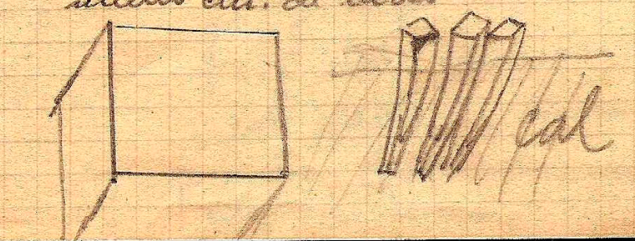

Fig. 5. Dibujo del mosaico en el diario de excavación de Alejandro Ramos Folqués. 


\section{Nueva lectura e interpretación}

El objetivo del trabajo es realizar una nueva edición del texto ya que el análisis directo y la revisión de las fotografías realizadas en el momento de su hallazgo (fig. 1) así como los diarios de excavación permiten hacer algunas correcciones significativas (fig. 5). ${ }^{21}$ El epígrafe se dispone en torno al cuadrado central que acoge la roseta y se distribuye de forma desigual: en un lado está escrita una única palabra con teselas rojas (ACOS) y en un segundo se superponen tres renglones de texto, que están separados entre sí por líneas de teselas rojas. Hay cinco puntos de lectura conflictivos: los inicios de tres líneas (2, 3 y 4), pues se ha perdido parte de la superficie del mosaico en esta zona que plantea problemas para reconocer las letras iniciales. No obstante, la distribución de las bandas en torno a la roseta central permite determinar que solo se ha perdido una letra (lín. 3) o que se conservan de forma parcial (lín. 2 y 4). El cuarto punto conflictivo se produce en la tercera de las líneas, ya que hay un trazo vertical de mayor tamaño que el resto de grafemas y de color diverso a los que le preceden se ha interpretado de dos formas diferentes: como una $i$ o como una separación. Finalmente, el quinto punto conflictivo lo ofrece la última línea, pues el estado de conservación del mosaico no solo afecta a la letra inicial sino a buena parte del final, habiéndose perdido entre tres y cinco letras, preservándose tan solo una parte del último grafema.

Las fotografías del momento de hallazgo (fig. 1), el dibujo realizado por Ramos en su diario de excavación (fig. 5) y los restos preservados en la actualidad (fig. 4) permiten observar que la primera letra de la segunda línea no puede ser sino B. Una B con un ojo superior sensiblemente más pequeño que el inferior que puede compararse con las empleadas en el bronce de Lascuta $\left(E L R H \mathrm{U} 1=\right.$ CIL I $\mathrm{I}^{2} 614=\mathrm{II}$ 5041) y en la tabula Contrebiensis $($ ELRH C9 = $C I L I^{2}$ 2951a). Pero la mayor dificultad para aceptar esta identificación es el resultado: una secuencia BL impropia del ibérico. Untermann solo indica una crux mientras que Gómez Pallarés y Corell se inclinan por reconocer O, por su parte, Siles reconstruye [BE]. Ramos y Uroz 1992, que ofrecen una lectura más precisa que la que había dado Ramos en la editio princeps, sí advierten la presencia de B ante $\mathrm{L}$.

21 La fotografía del descubrimiento fue publicada por Ramos 1975, Lám. LXXII fig. 3, y 1983, 98. Realizamos la autopsia el 27-7-2009, cuando el mosaico estaba desmontado en partes antes de realizar la última restauración de este pavimento. Hemos podido utilizar esta documentación inédita gracias a la amabilidad de A. M. Ronda Femenia, que ha dedicado su Tesis Doctoral a estudiar los diarios de excavación de Alejandro Ramos. 
Lo más razonable es leer la secuencia BL, impropia del ibérico pero que ahora cuenta con el paralelo de Bastobles, un nombre personal ibérico documentado en la recién editada inscripción latina de los cuatorviros de Oceja (Ferrer et al. 2018). Los editores reconocen un primer formante basto (MLH III-1, $\$ 7.28$; Rodríguez $\left.2014 \mathrm{n}^{\circ} 29\right)$ y un segundo elemento -bles, inédito pero que con toda probabilidad es una variante del ampliamente atestiguado formante beles (MLH III-1, $\$ 7.31$; Rodríguez 2014, $\mathrm{n}^{\circ} 34$ ), que no saben si explicar como un error de escritura o una peculiaridad fonética. Lo más plausible es que sea lo primero pues la secuencia fonética muta cum liquida no está atestiguada en ibérico (Quintanilla 1998, 34-39). Además, el formante beles está bien documentado en nombres personales atestiguados en inscripciones latinas y bles solo aparece en Oceja, por lo que es más probable que sea un error de escritura que una variante y lo mismo se puede decir respecto a $b l s$ y bels, aunque de este último hay menos testimonios (tabla 1). En definitiva, bles y bls no serían variantes de beles y bels sino errores de escritura y debería desarrollase: Bastob(e)les en Oceja y B(e)lsailacos en La Alcudia. Puede especularse con la posibilidad de que el scriptor hubiese cometido el error por influencia del semisilabario ibérico, en el que la sílaba be es representada por un solo signo.

\begin{tabular}{|c|c|c|}
\hline Formante onomástico & Signario & Inscripciones latinas \\
\hline $\begin{array}{l}\text { beles (MLH III-1 } \$ \\
7.31)\end{array}$ & beleś & $\begin{array}{l}\text { BELES: } \\
\left.\text { Beles (CIL I }{ }^{2} 709\right) \\
\text { Belesiar (HEp 16, 446) } \\
\text { Belestice (HEp 5, 465) } \\
\text { Bileseton (CIL II 3537) } \\
\left.\text { Estopeles (CIL I }{ }^{2} 709\right) \\
\text { Laurbeles (IRC II 83) } \\
\text { Neitinbeles (CIL II 6144) } \\
\left.\text { Ordumeles (CIL I I }{ }^{2} 709\right) \\
\left.\text { Turtumelis (CIL I }{ }^{2} 709\right) \\
\left.\text { Vmarbeles (CIL I }{ }^{2} 709\right) \\
\text { Bastobles (Oceja) }\end{array}$ \\
\hline $\begin{array}{l}\text { beles (MLH III-1 } \$ \\
7.32)\end{array}$ & bels & $\begin{array}{l}\text { BELS: } \\
\left.\text { Adimels (CIL I } \mathrm{I}^{2} 709\right) \\
\left.\text { Bennabels (CIL I } \mathrm{I}^{2} 709\right) \\
\left.\text { Sanibelser (CIL I } \mathrm{I}^{2} 709\right) \\
\text { Blsailacos (La Alcudia) }\end{array}$ \\
\hline
\end{tabular}

Tabla 1. Testimonios de los formantes onomásticos beles y bels. 
La influencia del sistema semisilábico para explicar que no se haya escrito la vocal $\mathrm{E}$ - pues estaba en cierto modo implícita en la letra B y en la escritura ibérica solo se empleaba un signo para representar esta sílaba- puede hacerse extensible a la última línea. En ella también aparece una secuencia de muta cum liquida impropia del ibérico: ESCRAD (Ballester 2001, 481), pero es muy probable que, como señalan Siles y Untermann, haya que suponer una $\mathrm{E}$ tras C y reconocer el formante esker ( $M L H$ III-1, $\$ 7.64$ ). Plausiblemente se dejo de escribir E porque era la vocal de apoyo para nombrar la letra y también por la influencia del sistema ibérico en la persona que redactó el texto. ${ }^{22}$

El segundo punto conflictivo lo representa la letra perdida al comienzo de la tercera línea, pero teniendo en cuenta que le sigue ELS y la restitución de $\mathrm{B}(\mathrm{E}) \mathrm{LS}$ al comienzo de la segunda, lo más plausible es suponer que la letra perdida sea B, como ya había considerado Siles 1978, solución que ahora también se apoya en la nueva lectura de la segunda línea. El tercer punto conflictivo está en este mismo renglón y puede reconocerse como una separación o como una I longa, siendo lo más plausible lo primero pues tras ella aparece una tesela azul a media altura de la caja de escritura que ya parece estar marcando la división de palabras (fig. 6).

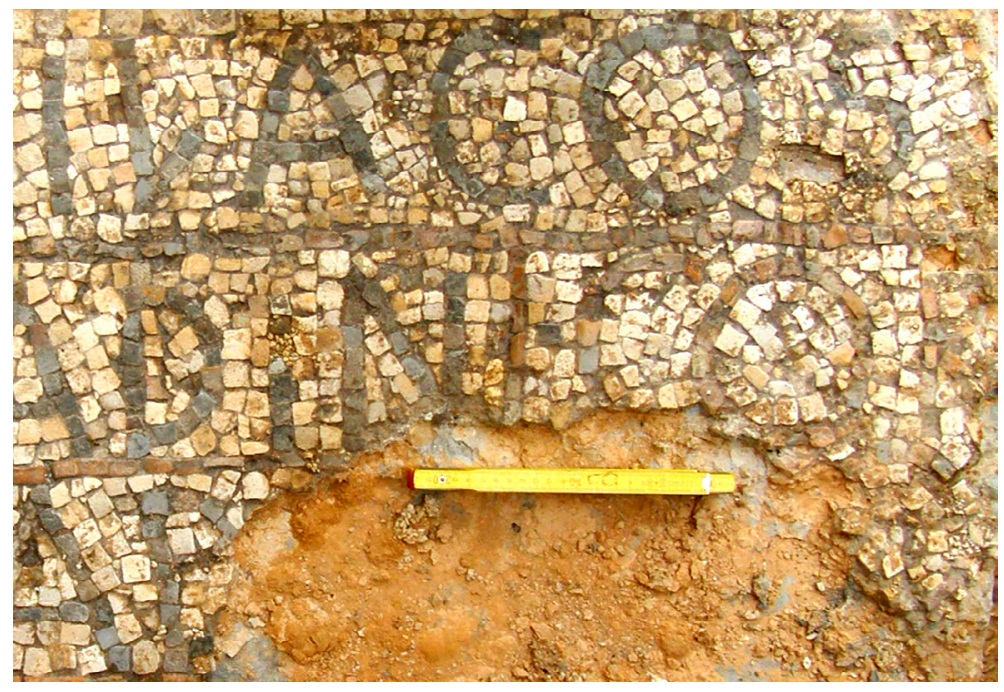

Fig. 6. Fotografía de detalle de las líneas 2-4

(foto: autor).

22 Sobre el problema del nombre de las letras en latín: Gordon 1973. 
Los dos últimos problemas que plantea la lectio del epígrafe atañen al último renglón. De la primera letra solo se preserva parte de un trazo horizontal inferior, pero solo puede corresponder a una E, puesto que es incompatible con la forma de L que se emplea en el texto. El hecho de que no se haya conservado el extremo del trazo horizontal del centro se explica porque fuese más corto, como se puede apreciar en la E del tercer renglón. La segunda parte de esta línea está perdida, aunque es posible reconstruir $\mathrm{AD}$ [IN] como propuso Siles y al final aún se aprecian restos de la última letra, un trazo curvo, quizá $\mathrm{D}$ pero más probablemente $\mathrm{O}$. Esta es la nueva propuesta de lectura y el aparato crítico de este epígrafe:

\author{
ACOS \\ BLSAILACOS \\ [.]ELSADINI $\cdot$ COR \\ ESCRAD[-c.3-5-]O
}

Lín. 1. [---]ACOS, Gómez Pallarès 1991. Lín. 2. Satu(?) Acos, D’Ors 1960; [---]L SAILACOS, Ramos 1975; [BE]LSAILACOS, Siles 1978; [---]L · SAILACOS[---], Rabanal y Abascal 1985; +LSAILACOS[---], MLH; [---]LSAILACOS, Gómez Pallarès 1991; [---]BLSAILACOS, Ramos y Uroz 1992; [O?] LSAILACOS, ECIMH; OLSAILACOS, Corell 1999. Lín. 3. et Sabini Cor(nelii), D’Ors 1960; [---]EL SADINICOR, Ramos 1975; [B]ELSADINICOR, Siles 1978; [---]ELSADINI · COR [---], Rabanal y Abascal 1985; [---]ELSADINI - COR[---] o [---]ELSADIN · COR [---], MLH; [---]ELSADINI · COR, Gómez Pallarès 1991; [---]EL SADINCOR, Ramos y Uroz 1992; [B?]ELSADIN - COR, ECIMH, Corell 1999. Lín. 4. scrad(?) [---], D’Ors 1960; [---]SCRAD[---], Ramos 1975, Gómez Pallarès 1991; [I]SCRAD[IN][--], Siles 1978; [---]SCCRAD[---], Rabanal y Abascal 1985; ESCRAD[---]+[---], MLH; [---]ESCRAD[---] O, Ramos y Uroz 1992; [E-?]SCRAḌ[-c.4-6-], ECIMH; ESCRAḌ[-c.4-], Corell 1999.

Bibl.: Siles 1978; Rabanal y Abascal 1985, n. 9 96; MLH III-2, G.12.4; Gómez Pallarès 1991, 81-82; Ramos y Uroz 1992, 97-98; ECIMH, A1; Corell 1999, n. ${ }^{\circ} 24,2012$, n. ${ }^{\circ}$ 24; Jaeggi 1999, 170-171; Notermans 2007, F36.

La lectura con la inclusión de las vocales no escritas y las reconstrucciones propuestas por Siles es la siguiente: ACOS / B(E)LSAILACOS / [B]ELSADINI - COR / ESC(E)RAD[IN -c.3-5-]O. Respecto a la interpretación del texto, hay que destacar cómo se repiten varios elementos: ACOS, B(E)LS [B]ELS y ADIN - AD[IN]. Bels y adin son formantes onomásticos bien documentados, también esker, pero no así acos, aunque aquí parece funcionar como tal. Solo hay una interpunción en el texto pero es posible que haya que realizar otras cesuras, de modo que puede segmentarse: Acos B(e)lsailacos, que puede compararse con Beles Vmarbeles $f$. (fórmula onomástica documentada en el bronce de Áscoli, CIL I ${ }^{2}$ 709), o Acosb(e)ls Ailacos, una fórmula en la 
que padre e hijo tienen nombres compuestos por dos formantes y comparten uno de ellos, como sucede con Ilurtibas Bilustibas $f$., Balciadin Balcibil $f$., Albennes Belennes $f$., Sosinaden Sosinasae $f$. y Sosimilus Sosinasae $f$., todos ellos atestiguados en el bronce de Áscoli, aunque en estos el elemento compartido siempre ocupa idéntica posición. Existe el problema de que acos no está documentado como formante onomástico y tampoco el segmento ail, que pudiera también interpretarse como sail o aila. Siles 1978, compara el nombre con beleśaiŕ (F.7.1), pudiéndose identificar śaiŕ, con elisión de la vibrante ante la lateral y, por tanto, un antropónimo trimembre. ${ }^{23}$

En la tercera línea se puede reconstruir un nombre personal Belsadin con dos formantes bien conocidos, bels y adin. Tras la interpunción se lee COR, que pudiera ser un término del léxico común o un formante onomástico abreviado como kori y koro (MLH III-1, $\$ 7.78$ y 79), primer elemento de un nombre personal que habría perdido la vocal final por su encuentro con esker (uid. Quintanilla 1998, 133). Tampoco puede excluirse reconocer un nombre esc(e)rad [in] en la última línea, lo que dejaría a COR aislado.

En definitiva, se reconocen tres formantes onomásticos ibéricos bien documentados: bels, adin y esker y es plausible que también lo sea acos. De ellos, acos, adin y bels aparecen dos veces y es probable que se deba a que los personajes mencionados en el epígrafe sean padres e hijos, pues en el bronce de Áscoli está documentado que vástago y progenitor pueden compartir un mismo formante. Sin embargo, no es fácil determinar cómo deben agruparse dichos formantes y cuántos y cuáles son los antropónimos que aparecen en el texto, tarea que complica el hecho de que solo haya una interpunción. Tampoco puede identificarse pospuesto a ellos ningún sufijo propio de la lengua ibérica y solo se atestigua un final - $i$ tras Belsadin, que Untermann interpreta como un genitivo latino. $\mathrm{Si}$, efectivamente, es una desinencia de genitivo, marcaría una dependencia del nombre anterior respecto a Belsadin, quizá filiación o de otro tipo, que quizá esté indicando el término COR. ${ }^{24} \mathrm{Si}$ Belsadini presenta una desinencia latina de genitivo, como sugería Untermman, estaría adaptado a la segunda declinación, algo que contrasta con lo que se conoce en otras inscripciones pues los antropónimos ibéricos, que habitualmente terminan en consonante, suelen adaptarse a la tercera declinación (Albertos 1982). Si se acepta que la $i$ longa representa una vocal larga y que es un genitivo debería aceptarse un nominativo Belsadinius, un gentilicio formado con

23 Faria 2007, 175, que tampoco excluye sail, cf. Rodríguez 2014, nº 112.

24 La existencia de un sufijo - $i$ en ibérico es dudosa, $c f . M L H$ III-1, $\$ 524$. 
el añadido del sufijo latino -ius a un nombre personal indígena, un tipo de construcciones que están testimoniadas en la Hispania Indoeuropea pero no en la onomástica ibérica.

\section{Conclusiones}

La revisión de la inscripción permite identificar como una B la letra parcialmente borrada al comienzo de la segunda línea. Esta lectura arroja una secuencia - $b l$ - impropia de la lengua ibérica, pero que ahora encuentra un paralelo en uno de los nombres personales ibéricos que aparecen en la recientemente publicada inscripción rupestre de Oceja: Bastobles. Se propone que bls y bles en La Alcudia y en Oceja, respectivamente, no sean variantes únicas de los bien atestiguados formantes onomásticos bels y beles sino que, por influencia de la semisilabario ibérico, se haya elidido la vocal que está implícita en la oclusiva, en este caso E. En el texto de La Alcudia también sucede en la última línea: escr, que debe interpretarse como el formante onomástico esc(e)r. No se trata de una norma ortográfica, pues no se cumple en la segunda línea si se acepta reconstruir [b]eles en su inicio. Este tipo de 'notación silábica' está bien documentada en la epigrafía latina republicana de Praeneste. ${ }^{25}$ Pero en el caso de la inscripción de La Alcudia parece más bien un lapsus calami de alguien acostumbrado a una escritura en la que las sílabas formadas por una oclusiva y una vocal se representan con un único signo. Es plausible que el fallo se produzca con $\mathrm{B}$ y $\mathrm{C}$ precisamente porque en el nombre nombre latino de estas letras (be y ce) está implícita la vocal $e$.

En el texto, como ya habían señalado los editores anteriores, aparecen varios nombres personales ibéricos. Se reiteran varios formantes onomásticos: $\mathrm{ADIN}$, en la segunda línea y que es plausible reconstruir en la tercera; BELS, que puede identificarse ahora en la segunda línea y que es probable reconstruir en la tercera; y ACOS, que probablemente es un formante onomástico como también es posible interpretarlo en la leyenda monetal que aparece en dracmas de imitación emporitana: akos (MLH I A.6.02; De Hoz 2011, 429). La reiteración de estos tres elementos hace pensar que se trata de varias fórmulas onomásticas compuestas por un nombre personal más el patronímico, pues en el bronce de Áscoli aparecen varios padres e hijos que comparten uno de los dos formantes onomásticos que componen sus nombres. La consecución de lo que bien pudieran ser dos fórmulas onomásticas compuestas por un 
nombre personal y el nombre del padre. Sin embargo, no es fácil determinar cuáles son los nombres personales, pues en todo el texto solo hay una única interpunción.

En las inscripciones musivas latinas coetáneas, cuando aparecen dos o más fórmulas onomásticas, son las de los miembros de un colegio de magistrados. La norma es que se indique el cargo preciso que ocupan (duoviros o ediles) y también la acción edilicia que acometieron. ${ }^{26}$ Las inscripciones musivas oscas también rememoran obras realizadas por magistrados (ImIt, Cumae 2 y Pompei 23), pero solo en el ejemplar pompeyano del templo de Dionisio se recoge el nombre de los dos miembros de un colegio (dos ediles). ${ }^{27}$

Los investigadores que han estudiado la inscripción de La Alcudia aceptan que se trata de un grupo de nombres personales. Gómez Pallarés (ECIMH, A1) considera que serían los comitentes y Lara 2007, 164, que fuesen miembros de un collegium de comerciantes. Esta hipótesis también se ha planteado recientemente para likine, que aparece en los dos pavimentos inscritos del valle del Ebro, a partir de su identificación con el liberto Licinus de la inscripción musiva de La Cabañeta $(A E 2001,1237)$, en la que se le califica de magister, presumiblemente de un collegium dedicado al comercio, de modo y manera que los lugares en los que aparecen ambos pavimentos con inscripciones ibéricas no serían viviendas, como se ha considerado tradicionalmente, sino stationes..$^{28}$ En la inscripción ilicitana aparecen varios nombres personales, es posible que deban agruparse en dos o más fórmulas onomásticas. Los paralelos que ofrece la epigrafía latina musiva inducen a pensar que son magistrados y que el texto es edilicio, pero el mosaico de La Alcudia se ubica en un espacio doméstico y no un edificio público como los paralelos latinos (principalmente templos y termas), ni puede reconocerse ningún término del léxico común que pudiera interpretarse como el verbo de la acción o bien como la magis-

26 CIL I 3368 (Luna), EE VIII 829 (Septempeda), CIL I 765 (Montonio al Vomano), AE 2004, 489 (Superaequum), CIL I² 1496 (Tibur), CIL I² 2948 (Capua), CIL I² 2542 (Crotona).

27 ImIt, Pompei 14. El ejemplar de Schiavi d’Abruzzo es de interpretación más incierta (ImIt, Teruentum 36). Un ejemplar muy fragmentario y ahora perdido de Falerii Novi recoge una inscripción falisca en la que se menciona el nombre de dos pretores (CIL XI 3156a).

28 Beltrán 2016. En Hispania hay dos epígrafes musivos republicanos en los que aparecen mencionados magistri de collegia: Mazarrón (ECIMH MU2) y el citado de La Cabañeta ( $A E$ 2001, 1237; ELRH C105), este segundo se ubica en unos almacenes mientras que es desconocido en qué tipo de edificio se emplazaba el primero. 
tratura de los personajes, aunque pudieran ubicarse al final de la última línea, donde la inscripción está perdida.

Un último aspecto a comentar es la clasificación lingüística del texto. El epígrafe está escrito en alfabeto latino, pero desde un inicio se ha aceptado que recogía nombres personales ibéricos y que, incluso, el texto podía clasificarse como ibérico dado que solo hay antropónimos vernáculos y ningún término ni desinencia latinos. Untermann lo editó en $M L H$, pero reconocía la posibilidad de que el final en - $i$ que precede a la única interpunción que hay en el epígrafe pueda ser una desinencia de genitivo latina. Todos los términos reconocibles en el texto son formantes onomásticos ibéricos, también lo parece acos, por lo que no hay ningún término del léxico común ni tampoco ningún sufijo, ya que la existencia de un sufijo ibérico - $i$ es incierta. Precisamente, el único argumento para clasificar el texto como latino es dicho final en - $i$, pues el resto de términos pueden reconocerse como nombres personales ibéricos, quizá nombres de padres e hijos como ya hemos visto. En tal caso debe subrayarse que en la primera parte, en la que existen dos posibles segmentaciones: Acos $B(e) l s a i l a c o s$ y Acosb(e)ls Ailacos, el segundo nombre no presenta desinencia de genitivo, como también sucede en este tipo de fórmulas onomásticas que aparecen en otras inscripciones latinas que recogen nombres personales ibéricos (bronce de Áscoli, bronce de Contrebia y la inscripción rupestre de Oceja), pero tampoco el término latino $f$ (ilius), que sí se incluye en los mencionados epígrafes.

La adaptación del nombre personal ibérico Belsadin (Belsadini en el epígrafe) a la segunda declinación es excepcional, porque mayoritariamente los antropónimos ibéricos, en su mayoría acabados en consonante, se adaptan a la tercera declinación latina. Existe la posibilidad de que esté adaptado a la tercera declinación si se clasifica como un dativo. En tal caso, el primer segmento del epígrafe podría interpretarse como los nombres personales de dos individuos o de un único (NP + patronímico) que hacen algo en favor de Belsadin. Entre la epigrafía musiva latina coetánea hay algún paralelo para esta estructura sintáctica, se trata del texto religioso de Cerro Gallufo (Cartagena): M. Aquini(us) M. l. Andro / Ioui Statori de sua p(ecunia) qur(auit) / l(ibens) $m$ (erito). ${ }^{29}$ Es sugerente la posibilidad de interpretar el segmento COR como abreviatura de coerauit, con el diptongo oe en lugar de $u$, algo común en la epigrafía latina republicana, pero en los índices de CIL I, p. 1212, 
no hay paralelos para tal apócope, atestiguándose co, coe, coer, coera, coerau, coeraue, coerauer y también coir por coirauit/coirauerunt, pero nunca cor. Si se acepta la clasificación de Belsadini como dativo difícilmente pudiera ser otra cosa que una divinidad. Por desgracia, solo se conocen tres teónimos ibéricos cuya clasificación como divinidades es segura por aparecer en inscripciones religiosas romanas: Betatun, en una inscripción de Fuente del Rey (Granada), betun-atun (Corzo et al. 2007); Salaeco, en un epígrafe de Portmán, śalai-ko (Velaza 2015); y Sertundo, nueva lectura para un epígrafe sobre un ara de Susqueda (Gerona), sertun-do (IRC III 10; Vidal 2016). El primero carece de una desinencia latina de dativo y no es seguro si la tienen los otros dos, aunque pudieran estar adaptados a la segunda declinación.

En conclusión, los paralelos latinos ofrecen la posibilidad de clasificar el texto como un epígrafe edilicio, en tal caso la consecución de nombres personales serían las denominaciones de varios magistrados que encargaron la obra, o como una dedicatoria, en tal caso cupiera clasificar Belsadini como un teónimo ibérico con desinencia latina de dativo. Lamentablemente, el edificio en el que se emplazaba el mosaico solo se conservaba de forma parcial y no es bien conocido, aunque se ha clasificado como una vivienda, algo que no encaja bien con ninguna de las propuestas, ni la de interpretarlo como un texto edilicio, que aparecen en termas y templos, ni tampoco la de que sea la dedicatoria de un templo.

Agradecimientos: Este trabajo ha sido posible gracias a la colaboración del equipo de la fundación La Alcudia y especialmente a A. Ronda Femenia.

\section{|}

Abad 1987: L. Abad, “En torno a dos mosaicos Ilicitanos: el 'helenístico' y el de conchas marinas", CUPAUAM 13-14, 1987, 97-105.

Abad 2004: L. Abad, "La Alcudia ibérica. En busca de la ciudad perdida", en: Iberia, Hispania, Spania. Una mirada desde Ilici, Alicante 2004, 69-78.

Albertos 1982: M. L. Albertos, “Onomastique personnelle indigène de la Péninsule Ibérique sous la domination romaine", en: ANRW 29.2, 1982, 853-892.

Ballester 2001: X. Ballester, "El substrato de la lengua ibérica en la Península Ibérica", en: Congrés Internacional de Toponímia i Onomàstica Catalanes, Valencia 2001, 459-288.

Beltrán 2016: F. Beltrán, “Sedes colegiales indígenas de fecha republicana en Caminreal y Andelo?", en: O. Rodríguez, N. Tran y B. Soler (eds.), Los espacios de reunión de las asociaciones romanas. Diálogos desde la arqueología y la historia, en homenaje a Bertrand Goffaux, Sevilla 2016, 331-344.

Broise y Jolivet 2004: H. Broise y V. Jolivet, Musarna II. Les bains hellénistiques, Roma 2004. 
Ignacio Simón Cornago

Bruneau 1972: Ph. Bruneau, Délos. Les Mosä̈ques, París 1972.

CNH: L. Villaronga, Corpus nummum Hispaniae ante Augusti aetatem, Madrid 1994.

Coarelli 1976: F. Coarelli, "Un elmo con iscrizione latina arcaica al Museo di Cremona", en: Mélanges offerts à Jacques Heurgon, Roma 1976, 157-179.

Corell 1999: J. Corell, Inscripcions romanes d'Ilici, Lucentum, Allon, Dianium i el seus territoris, Valencia 1999.

Corell 2012: J. Corell, Inscripcions romanes del País Valencià. Ilici, Lucentum, Allon, Dianium i el seus territoris, Valencia 2012.

Corzo et al. 2007: S. Corzo, M. Pastor, A. U. Stylow y J. Untermann, “Betatun, la primera divinidad ibérica identificada”, PalHisp 7, 2007, 251-262.

Cristofani 1969: M. Cristofani, “La tomba del 'Tifone'. Cultura e società di Tarquinia in età tardo etrusca”, Atti della Accademia nazionale dei Lincei. Classe di scienze morali, storiche e filologiche, Memorie VIII, 14, 1969, 213-256.

De Hoz 2011: J. De Hoz, Historia lingüística de la Península Ibérica en la Antigüedad II. El mundo ibérico prerromano y la indoeuropeización, Madrid 2011.

De Simone 2001: A. De Simone, "Una data consolare in un pavimento a Pompei”, en: AISCOM VII, Ravena 2001, 247-253.

Dell'Aglio y Masiello 2004: A. Dell'Aglio y L. Masiello, "Recenti rivenimenti musivi a Taranto", en: Apparati musivi antichi nellarea del Mediterraneo, Palermo 2004, 49-59.

D’Ors 1960: A. D’Ors, “Miscelánea epigráfica”, Emerita 28, 1960, 327-330.

ECIMH: J. Gómez, Edición y comentario de las inscripciones sobre mosaico de Hispania. Inscripciones no cristianas, Roma 1997.

ELRH: B. Díaz, Epigrafía latina republicana de Hispania, Barcelona 2008.

IRC: G. Fabre, M. Mayer y I. Rodà, Inscriptions romaines de Catalogne I-V, París 1984-2002.

Jaeggi 1999: O. Jaeggi, Der Hellenismus auf der Iberische Halbinsel, Mainz 1999.

Faria 1995: A. M. Faria, "Novas achegas para o estudo da onomástica ibérica e turdetana", Vipasca 4, 1995, 79-88.

Faria 2007: A. M. Faria, “Crónica de onomástica paleo-hispânica (13)”, RPA 10.2, 2007, 161-187.

Faria 2018: A. M. Faria, “Crónica de onomástica paleo-hispânica (26)”, RPA 21, 2018, 115-130.

Fernández 2003: A. Fernández, "Adopción de las técnicas pictóricas y musivarias entre las sociedades íberas", en: L. Abad (ed.), De Hiberia in Hispania. La adaptación de las sociedades ibéricas a los modelos romanos, Alicante 2003, 209-239.

Fernández-Galiano 1982: D. Fernández-Galiano, "New light on the origins of floor mosaics", The Antiquaries Journal 62, 1982, 235-244.

Ferrer et al. 2018: J. Ferrer, J. Velaza y O. Olesti, "Nuevas inscripciones rupestres latinas de Oceja y los IIIIviri ibéricos de Iulia Lybica", DHA 44.1, 2018, 169-195.

Gómez Pallarès 1991: J. Gómez Pallarès, "Nombres de artistas en inscripciones musivas latinas e ibéricas de Hispania”, Epigraphica 53, 1991, 59-96.

Gordon 1973: A. E. Gordon, The letter names of the Latin alphabet, Londres 1973.

ImIt: M. H. Crawford (ed.), Imagines italicae. A corpus of Italic inscriptions, Londres 2011.

Lara 2007: G. Lara, "Nuevos datos para la contextualización del mosaico helenístico de La Alcudia (Elche, Alicante)", en: L. Abad y J. A. Soler (eds), Arte ibérico en la España mediterránea, Alicante 2007, 156-166. 
Mezquíriz 1992: M. A. Mezquíriz, "Inscripción ibérica de Andelos (Mendigorría, Navarra)", en: Estudios de Arqueología ibérica y romana. Homenaje a Enrique Pla Ballester, Valencia 1992, 347-349.

MLH: J. Untermann, Monumenta Linguarum Hispanicarum, Wiesbaden 1975-1997.

Notermans 2007: A. Notermans, Sprekende mozaïeken. Functie en betekenis van teksten op Romeinse vloermozaïeken, Nijmegen 2007.

Oliver 1966: R. P. Oliver, “Apex and Sicilicus”, AJPh 87.2, 1966, 129-170.

Quintanilla 1998: A. Quintanilla, Estudios de fonología ibérica, Vitoria 1998.

Rabanal y Abascal 1985: M. A. Rabanal y J. M. Abascal, "Inscripciones romanas de la provincia de Alicante", Lucentum 4, 1985, 191-244.

Rabanal y Abascal 1986: M. A. Rabanal y J. M. Abascal, “Dos nuevas inscripciones y algunas correcciones a la epigrafía romana de la provincia de Alicante”, Lucentum 5, 1986, 196 174.

Ramallo 1985: S. F. Ramallo Asensio, Mosaicos romanos de Carthago Nova (Hispania Citerior), Murcia 1985, 62-63.

Ramos 1969: R. Ramos Fernández, "Inscripciones ibéricas de La Alcudia (Elche)”, APL 12, 1969, 169-177.

Ramos 1975: R. Ramos Fernández, La ciudad romana de Illici. Estudio Arqueológico, Alicante 1975.

Ramos 1983: R. Ramos Fernández, La Alcudia de Elche, Elche 1983.

Ramos 1983: R. Ramos Fernández, "Estratigrafía del sector 5-F de La Alcudia de Elche", Lucentum 2, 1983, 147-172.

Ramos Folqués 1975: A. Ramos Folqués, “Un mosaico helenístico en La Alcudia de Elche”, APL $14,1975,69-81$.

Ramos y Uroz 1992: R. Ramos y J. Uroz, “Ilici”, Dialoghi di Archeologia 10, 1992, 95-104.

Rodríguez 2014: J. Rodríguez Ramos, "Nuevo Índice Crítico de formantes de compuestos de tipo onomástico íberos", ArqueoWeb 15, 2014, 81-238.

Ruiz 2001: E. Ruiz Roig, Los mosaicos de Illici y del Portus Illicitanus, Valencia 2001.

Salcuni 2008: A. Salcuni, "Decorazioni pavimentali negli edifici templari di età repubblicana in Abruzzo", en: AISCOM XIII, Tívoli 2008, 277-288.

Salcuni 2013: A. Salcuni, Pitture parietali e pavimenti decorati di epoca romana in Abruzzo, Bonn 2013.

San Nicolás 2004: M. P. San Nicolás, "Mosaicos hispano-romanos con representación de murallas", en: L'Africa romana XV. Ai confini dell'Impero: contatti, scambi, conflitti, Roma 2004, 825-852.

Sarabia y Cañavete 2010: J. Sarabia y V. Cañavete, "La arquitectura doméstica romana en La Alcudia de Elche. La Domus 5-F”, Lucentum 28, 2010, 89-110.

Salzmann 1982: D. Salzmann, Untersuchungen zu den antiken Kieselmosaiken, Berlín 1982.

Siles 1978: J. Siles, “Einheimische Eigennamen auf einem hellenistischen Mosaik”, Beiträge zur Namenforschung 13, 1978, 331-340.

Simón 2015: I. Simón, “Treinta años de investigaciones sobre la inscripción musiva de Caminreal (Hispania Citerior; MLH E.7.1 = K.5.3)", PalHisp 15, 2015, 87-127.

Simón 2018: I. Simón, "Las inscripciones musivas en Italia y el Mediterráneo Occidental durante los siglos III-I a.C.", en: F. Beltrán y B. Díaz (eds.), El nacimiento de las culturas epigráficas en el Occidente Mediterráneo. Modelos romanos y desarrollos locales (III-I a.E.), Madrid 2018, 253-287. 
Simón 2019: I. Simón, "Lenguas vernáculas de Hispania escritas en alfabeto latino: un episodio particular de la latinización", Athenaeum 107.1, 2019, 55-93.

Tammisto 1997: A. Tammisto, Birds in mosaics. A Study on the Representation of Birds in Hellenistic and Romano-Campanian Tessellated Mosaics on the Early Augustan Age, Roma 1997.

Tendero y Ronda 2014: M. Tendero y A. M. Ronda, "La ciudad romana de Ilici (L'Alcúdia de Elche, Alicante)", en: M. H. Olcina (ed.), Ciudades Romanas Valencianas, Alicante 2014, 226-249.

Untermann 1993-94: J. Untermann, “Comentario a la inscripción musiva de Andelos”, TAN 11, 1993-94, 127-129.

Vallejo 2004: J. M. Vallejo, Antroponimia indígena de la Lusitania romana, Vitoria 2004.

Vassal 2006: V. Vassal, Les pavements d’opus signinum. Technique, décor, fonction architecturale, Oxford 2006.

Velaza 2015: J. Velaza, “Salaeco: un teónimo ibérico”, ZPE 194, 2015, 290-291.

Vicente et al. 1989: J. Vicente, J. Martín, C. Escriche, A. I. Herce y M. P. Punter, “Un pavimento musivo de opus signinum con epígrafe ibérico", en: Mosaicos romanos. In memoriam Manuel Fernández Galiano, Madrid 1989, 11-42.

Vidal 2016: J. C. Vidal, “Interpretació ibèrica de dos teònims preromans del nord-est peninsular”, RAP 26, 2016, 195-204.

Vine 1993: B. Vine, Studies in Archaic Latin Inscriptions, Innsbruck 1993. 